\title{
Inventory Model with Price-Dependent Demand Rate and No Shortages: An Interval-Valued Linear Fractional Programming Approach
}

\author{
Dr. Pavan Kumar \\ Assistant Professor, Department of Mathematics, \\ Faculty of Science and Technology, \\ IIS University, Jaipur-302020, India.
}

\begin{abstract}
In this paper, an interval-valued inventory optimization model is proposed. The model involves the pricedependent demand and no shortages. The input data for this model are not fixed, but vary in some real bounded intervals. The aim is to determine the optimal order quantity, maximizing the total profit and minimizing the holding cost subjecting to three constraints: budget constraint, space constraint, and budgetary constraint on ordering cost of each item. We apply the linear fractional programming approach based on interval numbers. To apply this approach, a linear fractional programming problem is modeled with interval type uncertainty. This problem is further converted to an optimization problem with intervalvalued objective function having its bounds as linear fractional functions. Two numerical examples in crisp case and interval-valued case are solved to illustrate the proposed approach.
\end{abstract}

\section{Keywords}

Inventory model, Price-dependent demand, Interval-valued function, Fractional programming.

\section{INTRODUCTION}

For many practical inventory problems, some parameters are uncertain. Therefore to obtain more realistic results, the uncertainty in parameters must be considered, and the corresponding uncertain optimization methods must be constructed. Some techniques have been developed to solve uncertain optimization problems. The fuzzy and stochastic approaches are normally applied to describe imprecise characteristics. In these two types of techniques, the membership function and probability distribution play important roles.

This is, however, practically difficult to specify a precise probability distribution or membership function for some uncertain parameter. Over the past few decades, the interval number programming approach has been developed to deal with such type of problems in which the bounds of the uncertain parameters are only required. An optimization problem with interval coefficients is referred as the interval-valued optimization problem. In this case, the coefficients are assumed as closed intervals.

Charnes et al (1977) proposed a concept to solve the linear programming problems in which the constraints are considered as closed intervals. Steuer (1981) developed some algorithms to solve the linear programming problems with interval objective function coefficients. Alefeld and Herzberger (1983) introduced the concept of computations on interval-valued numbers. Ishibuchi and Tanaka (1990), and then Chinneck and Ramadan (2000) proposed the linear

DOI : 10.5121/oraj.2015.2402 
Operations Research and Applications : An International Journal (ORAJ), Vol.2, No.4, November 2015

programming problem (LPP) with interval coefficients in objective function. Tong (1994) studied the interval number and fuzzy number linear programming, and considered the case in which the coefficients of the objective function and constraints are all interval numbers. The possible interval-solution was obtained by taking the maximum value range and minimum value range inequalities as constraint conditions.

Moore and Lodwick (2003) further extended the theory of interval numbers and fuzzy numbers. Mráz (1998) proposed an approach to determine the exact bounds of optimal values in LP with interval coefficients. In the interval number programming, the objective function and constraints may not always be linear, but are often nonlinear. Levin (1999) introduced the nonlinear optimization under interval uncertainty. Sengupta et al (2001) studied the Interpretation of inequality constraints involving interval coefficients and a solution to interval linear programming.

The optimality conditions of Karush-Kuhn-Tucker (KKT) possess a significant role in optimization theory . In literature, various approaches to interval-valued optimization problems have been proposed in details, while few papers studied the KKT conditions for interval-valued optimization problems. Wu (2007) used the KKT conditions to solve an optimization problem with interval-valued objective function. Wu (2008a) studied the interval-valued nonlinear programming problems, and then Wu (2008b) \& (2010) established the Wolfe duality for optimization problems with interval-valued objective functions. Bhurjee and Panda (2012) suggested a technique to obtain an efficient solution of interval optimization problem.

In single objective optimization, the aim is to find the best solution which corresponds to the minimum or maximum value of a single objective function. As far as the applications of nonlinear programming problems are considered, a ratio of two functions is to be maximized or minimized under certain number of constraints. In some other applications, the objective function involves more than one ratio of functions. The problems of optimizing one or more ratios of functions are referred as fractional programming problems. Normally, most of the multi objective fractional programming problems are first converted into single objective fractional programming problems and then solved. The decision maker (DM) must altogether optimize these conflicting goals in a framework of fuzzy aspiration levels. Charnes and Cooper (1962) proposed that a linear fractional program with one ratio can be reduced to a linear program using a nonlinear variable transformation. Chanas and Kuchta (1996) generalized the solution concepts of the linear programming problem with interval coefficients in the objective function based on preference relations between intervals.

In the recent years, a kind of new applications of fractional programming was found in inventory optimization problems. Sadjadi et al (2005) proposed a fuzzy approach to solve a multi objective linear fractional inventory model. They considered two objective functions as fractional with two constraints: space capacity constraint, and budget constraint, and aimed to simultaneously maximize the profit ratio to holding cost and to minimize the back orders ratio to total ordered quantities. Carla Oliveira et al_(2007) presented an overview of multiple objective linear programming models with interval coefficients. Some applications of multi objective linear fractional programming in inventory were proposed by Toksarı (2008). Effati et al (2012) presented an approach to solve the interval-valued linear fractional programming problem.

Liu (2006) derived a computational method for the profit bounds of inventory model with interval demand and unit cost. Liu and Wang (2007) developed a method for numerical solution to interval quadratic programming. Liu (2008) discussed the posynomial geometric programming with interval exponents and coefficients. 
Operations Research and Applications : An International Journal (ORAJ), Vol.2, No.4, November 2015

Mishra (2007) studied some problems on approximations of functions in Banach Spaces, and then Deepmala (2014) studied the fixed point theorems for nonlinear contractions and its applications. Recently, Vandana and Sharma (2015) proposed an EPQ inventory model for non-instantaneous deteriorating item under trade credit policy. The concept of fuzzy was also used in case of fractional inventory model. Kumar and Dutta (2015) proposed a multi-objective linear fractional inventory model of multi-products with price-dependant demand rate in fuzzy environment.

This paper aims at extending the application of linear fractional programming to inventory optimization problems in interval uncertainty. We consider an interval-valued fractional objective function and interval-valued constraints for the proposed inventory problem. The paper is organized as follows. Some preliminaries are given in Section 2. In Section 3, the notations and assumptions are given. In Section 4, a linear fractional programming inventory problem is proposed in crisp sense. In Section 5, we formulate above problem considering interval uncertainty. Two numerical examples are solved in Section 6. Finally, we conclude in Section 7.

\section{PRELIMinaries}

Interval-Valued Number (Moore \& Lodwick (2003)):

Let $* \in\{+,-, ., /\}$ be a binary operation on real line $\mathfrak{R}$. Let $\mathrm{A}$ and $\mathrm{B}$ be two closed intervals. Then, a binary operation is defined as

$$
\mathrm{A} * \mathrm{~B}=\{\mathrm{a} * \mathrm{~b}: \mathrm{a} \in \mathrm{A}, \mathrm{b} \in \mathrm{B}\}
$$

In the case of division, it is assumed that $0 \notin \mathrm{B}$. The operations on intervals used in this paper are defined as:

a) $\mathrm{kA}= \begin{cases}{\left[k a_{L}, k a_{R}\right] ;} & \text { if } k \geq 0 \\ \left(k a_{R}, k a_{L}\right) ; & \text { if } k<0\end{cases}$

b) $\mathrm{A}+\mathrm{B}=\left[a_{L}, a_{R}\right]+\left[b_{L}, b_{R}\right]$

$$
=\left[a_{L}+b_{L}, a_{R}+b_{R}\right]
$$

c) $\mathrm{A}-\mathrm{B}=\left[a_{L}, a_{R}\right]-\left[b_{L}, b_{R}\right]$

$$
\begin{aligned}
& =\left[a_{L}, a_{R}\right]+\left[-b_{R},-b_{L}\right] \\
& =\left[a_{L}-b_{R}, a_{R}-b_{L}\right]
\end{aligned}
$$

d) $\mathrm{A} \times \mathrm{B}=\left[\min \left\{a_{L} b_{L}, a_{L} b_{R}, a_{R} b_{L}, a_{R} b_{R}\right\}, \max \left\{a_{L} b_{L}, a_{L} b_{R}, a_{R} b_{L}, a_{R} b_{R}\right\}\right]$

e) $\frac{\mathrm{A}}{\mathrm{B}}=\mathrm{A} \times \frac{1}{B}=\left[a_{L}, a_{R}\right] \times\left[\frac{1}{b_{R}}, \frac{1}{b_{L}}\right]$, provided $0 \notin\left[b_{L}, b_{R}\right]$,

Alternatively, division of interval numbers can be defined as $\frac{\mathrm{A}}{\mathrm{B}}=\frac{\left[a_{L}, a_{R}\right]}{\left[b_{L}, b_{R}\right]}=\left[\min \left\{\frac{a_{L}}{b_{L}}, \frac{a_{L}}{b_{R}}, \frac{a_{R}}{b_{L}}, \frac{a_{R}}{b_{R}}\right\}, \max \left\{\frac{a_{L}}{b_{L}}, \frac{a_{L}}{b_{R}}, \frac{a_{R}}{b_{L}}, \frac{a_{R}}{b_{R}}\right\}\right]$,

Alternatively, division of interval numbers can be defined as

$$
\frac{\mathrm{A}}{\mathrm{B}}=\frac{\left[a_{L}, a_{R}\right]}{\left[b_{L}, b_{R}\right]}=\left[\frac{a_{L}}{b_{R}}, \frac{a_{R}}{b_{L}}\right], \quad \text { where } 0 \notin \mathrm{B}, 0 \leq a_{L} \leq a_{R}, 0<b_{L} \leq b_{R} .
$$


Operations Research and Applications : An International Journal (ORAJ), Vol.2, No.4, November 2015

f) $A^{k}=\left[a_{L}, a_{R}\right]^{k}=\left\{\begin{array}{lr}{[1,1] ;} & \text { if } k=0 \\ {\left[a_{L}^{k}, a_{R}^{k}\right] ;} & \text { if } a_{L} \geq 0 \text { or k odd } \\ {\left[a_{R}^{k}, a_{L}^{k}\right] ;} & \text { if } a_{R} \leq 0 \text { or k even } \\ {\left[0, \max \left(a_{\mathrm{L}}^{\mathrm{k}}, a_{\mathrm{R}}^{\mathrm{k}}\right)\right] ; \text { if } a_{L} \leq 0 \leq a_{R},} & \text { and } k(>0) \text { is even }\end{array}\right.$

Interval-Valued Function: Let $\Re^{n}$ be an n-dimensional Euclidean space. Then a function

$$
f: \Re^{n} \rightarrow I
$$

is called an interval valued function (because $f(x)$ for each $\mathrm{x} \in \Re^{n}$ is a closed interval in $\mathfrak{R}$ ). Similar to interval notation, we denote the interval-valued function $f$ with

$$
f(x)=\left[f^{L}(x), f^{U}(x)\right]
$$

where for each $\mathrm{x} \in \mathfrak{R}^{n}, f^{L}(x), f^{U}(x)$ are real valued functions defined on $\mathfrak{R}^{n}$, called the lower and upper bounds of $f(x)$, and satisfy the condition:

$$
f^{L}(x) \leq f^{U}(x) .
$$

Proposition 1. Let $f$ be an interval valued function defined on $\mathfrak{R}^{n}$. Then $f$ is continuous at $\mathrm{c}$ $\in \mathfrak{R}^{n}$ if and only if $f^{L}$ and $f^{U}$ are continuous at c.

Definition 1. We define a linear fractional function $\mathrm{F}(\mathrm{x})$ as follows:

$$
\mathrm{F}(\mathrm{x})=\frac{c x+\alpha_{1}}{d x+\alpha_{2}}
$$

Where $\mathrm{x}=\left(x_{1}, x_{2}, \ldots, x_{n}\right)^{t} \in \mathfrak{R}^{n}, \mathrm{c}=\left(c_{1}, c_{2}, \ldots, c_{n}\right) \in \mathfrak{R}^{n}, \mathrm{~d}=\left(d_{1}, d_{2}, \ldots, d_{n}\right) \in \mathfrak{R}^{n}$, and $\alpha_{1}$, $\alpha_{2}$ are real scalars.

Definition 2. Let $F: V \rightarrow I$ be an interval-valued function, defined on a real vector space V. Now, we consider the following interval-valued optimization problem

(IVP) $\quad \operatorname{Min}_{\preccurlyeq} F(x)=\left[F_{L}(x), F_{U}(x)\right]$

Subject to $\mathrm{x} \in \mathrm{Y} \subseteq \mathrm{V}$,

where $\mathrm{Y}$ is the feasible set of problem (IVP) and $\preccurlyeq$ is the partial ordering on the set of integers.

Definition 3. Let $\preccurlyeq$ be a partial ordering on the set of integers. Then, for $a=\left[a^{L}, a^{U}\right]$, and $b=\left[b^{L}, b^{U}\right]$, we write

$$
\begin{aligned}
& a \leqslant b \\
& \text { if and only if } a^{L} \leq b^{L} \text { and } a^{U} \leq b^{U} .
\end{aligned}
$$

This means that $a$ is inferior to $b$ or $b$ is superior to $a$.

Definition 4. Let $\mathrm{F}: \mathrm{V} \rightarrow \mathrm{I}$ be an interval-valued function, defined on a real vector space $\mathrm{V}$, and let $\mathrm{Y}$ be a subset of $\mathrm{V}$. Suppose that we are going to maximize $\mathrm{F}$. We say that $\mathrm{F}(\bar{x})$ is a nondominated objective value if and only if there exists no $x(\neq \bar{x}) \in Y$ such that $F(\bar{x}) \prec F(x)$. 
Operations Research and Applications : An International Journal (ORAJ), Vol.2, No.4, November 2015

\section{Interval-Valued Linear Fractional Programming (IVLFP)}

$$
\begin{aligned}
& \text { Minimize } \quad \mathrm{f}(\mathrm{x})=\frac{c x+\alpha_{1}}{d x+\alpha_{2}} \\
& \text { Subject to } A x=b \\
& \mathrm{x} \geq 0
\end{aligned}
$$

Suppose $\mathrm{c}=\left(c_{1}, c_{2}, \ldots, c_{n}\right), \mathrm{d}=\left(d_{1}, d_{2}, \ldots, d_{n}\right)$, where $c_{j}, d_{j} \in I, j=1,2, \ldots, \mathrm{n}$.

Define $\quad c^{L}=\left(c_{1}^{L}, c_{2}^{L}, \ldots, c_{n}^{L}\right), c^{U}=\left(c_{1}^{U}, c_{2}^{U}, \ldots, c_{n}^{U}\right), d^{L}=\left(d_{1}^{L}, d_{2}^{L}, \ldots, d_{n}^{L}\right), d^{U}=$ $\left(d_{1}^{U}, d_{2}^{U}, \ldots, d_{n}^{U}\right)$, where $c_{j}^{L}, c_{j}^{U}, d_{j}^{L}, d_{j}^{L}$ are real scalars for $j=1,2, \ldots$, n. Also, $\alpha_{1}=\left[\alpha_{1}^{L}, \alpha_{1}^{U}\right]$, $\alpha_{2}=\left[\alpha_{2}^{L}, \alpha_{2}^{U}\right]$.

Then IVLFP (10) can be re-written as:

$$
\begin{array}{cc}
\text { Minimize } & \mathrm{f}(\mathrm{x})=\frac{p(x)}{q(x)} \\
\text { Subject to } \quad A x & =\mathrm{b} \\
\mathrm{x} & \geq 0,
\end{array}
$$

where $\mathrm{p}(\mathrm{x})$ and $\mathrm{q}(\mathrm{x})$ are interval-valued linear functions and are given by

$$
\begin{aligned}
& \mathrm{p}(\mathrm{x})=\left[p^{L}(x), p^{U}(x)\right]=\left[c^{L} x+\alpha_{1}^{L}, c^{U} x+\alpha_{1}^{U}\right] \\
& \mathrm{q}(\mathrm{x})=\left[q^{L}(x), q^{U}(x)\right]=\left[d^{L} x+\alpha_{2}^{L}, d^{U} x+\alpha_{2}^{U}\right] .
\end{aligned}
$$

Therefore, IVLFP (11) can re-written as

$$
\begin{aligned}
& \text { Minimize } \mathrm{f}(\mathrm{x})=\frac{\left[c^{L} x+\alpha_{1}^{L},\right.}{\left.d^{U} x+\alpha_{1}^{U}\right]} \\
& \text { Subject to } A x=\mathrm{b} \\
& \mathrm{x} \geq 0
\end{aligned}
$$

Further, using the concept of division of two interval-valued numbers, IVLFP (12) can re-written as

$$
\begin{array}{cl}
\text { Minimize } & \mathrm{f}(\mathrm{x})=\left[f^{L}(x), f^{U}(x)\right] \\
\text { Subject to } & A x=\mathrm{b} \\
& \mathrm{x} \geq 0
\end{array}
$$

where $f^{L}(x)$ and $f^{U}(x)$ are linear fractional functions.

Definition 5. (Wu (2008)) Let $x^{*}$ be a feasible solution of IVLFP (13). Then, $x^{*}$ is a nondominated solution of IVLFP (13) if there exist no feasible solution $\mathrm{x}$ such that $f(\mathrm{x}) \prec f\left(x^{*}\right)$. In this case, we say that $f\left(x^{*}\right)$ is the non-dominated objective value of $f$.

Corresponding to IVLFP (13)), consider the following optimization problem:

$$
\begin{gathered}
\text { Minimize } \mathrm{g}(\mathrm{x})=f^{L}(x)+f^{U}(x) \\
\text { Subject to } A x=\mathrm{b} \\
\mathrm{x} \geq 0
\end{gathered}
$$

To solve IVLFP (14), we use the following theorem (Wu (2008)).

Theorem. Wu (2008) If $x^{*}$ is an optimal solution of $\operatorname{IVLFP}(14)$, then $x^{*}$ is a non-dominated solution of IVLFP(13).

Proof. Let us refer to $\mathrm{Wu}(2008)$. 
Operations Research and Applications : An International Journal (ORAJ), Vol.2, No.4, November 2015

\title{
3. NOTATIONS AND ASSUMPTIONS
}

To develop the proposed model, we considered the following notations and assumptions:

\section{$\underline{\text { Notations }}$}

\author{
$\lambda$ : Fixed cost per order \\ $B$ : Maximum available budget for all products \\ $W$ : Maximum available space for all products \\ For $i^{\text {th }}$ product: $(i=1,2, \ldots, \mathrm{n})$ \\ $Q_{i} \quad$ : Ordering quantity of product $i$, \\ $h_{i} \quad$ : Holding cost per product per unit time for $\mathrm{i}^{\text {th }}$ product \\ $P_{i} \quad$ : Purchasing price of $\mathrm{i}^{\text {th }}$ product \\ $S_{i} \quad$ : Selling price of $\mathrm{i}^{\text {th }}$ product \\ $D_{i}$ : Demand quantity per unit time of $\mathrm{i}^{\text {th }}$ product \\ $f_{i} \quad$ : Space required per unit for $\mathrm{i}^{\text {th }}$ product \\ $O C_{i}$ : Ordering cost of $\mathrm{i}^{\text {th }}$ product.
}

\section{$\underline{\text { Assumptions }}$}

1. Multiple products are considered in this model.

2. Infinite time horizon is considered. Further, there is only one period in the cycle time.

3. Lead time is zero, and so rate of replenishment is infinite.

4. Holding cost is known and constant for each product.

5. Demand is taken here as inversely related to the selling price of the product, that is,

$$
D_{i}=D_{i}\left(S_{i}\right)=m_{1} S_{i}^{-m_{2}}
$$

where $m_{1}>0$ is a scaling constant, and $m_{2}>1$ is price-elasticity coefficient. For notational simplicity, we will use $D_{i}$ and $D_{i}\left(S_{i}\right)$ interchangeably.

6. Shortages are not allowed.

7. Purchase price is constant for each product, i.e. no discount is available.

\section{CRISP Linear FRACTIONAL PROGRAMMING INVENTORY PROBLEM}

A multi-product inventory model under resources constraints is introduced as a linear fractional programming problem. This model refers to a multi-product inventory problem, with limited capacity of warehouse and constraints on investment in inventories. For each product, we impose the constraint on ordering cost.

We practically observe the inventory models with more than one objective functions. These objectives may be in conflict with each other, or may not be. In such type of inventory models, the decision maker is interested to maximize or minimize two or more objectives simultaneously over a given set of decision variables. We call this model as linear fractional inventory model. Without loss of generality, we assume there is only one period in the cycle time. Then,

$$
\begin{aligned}
\text { Total Profit } & =\sum_{i=1}^{n}\left(S_{i}-P_{i}\right) Q_{i} \\
\text { Holding cost } & =\sum_{i=1}^{n} \frac{h_{i} Q_{i}}{2}
\end{aligned}
$$


Operations Research and Applications : An International Journal (ORAJ), Vol.2, No.4, November 2015

$$
\begin{aligned}
\text { Ordering cost } & =\sum_{i=1}^{n} \frac{\lambda D_{i}}{Q_{i}}=\sum_{i=1}^{n} \frac{\lambda m_{1} S_{i}^{-m_{2}}}{Q_{i}} \\
\text { Back ordered quantity } & =\sum_{i=1}^{n}\left(D_{i}-Q_{i}\right)=\sum_{i=1}^{n}\left(m_{1} S_{i}^{-m_{2}}-Q_{i}\right) \\
\text { Total ordered quantity } & =\sum_{i=1}^{n} Q_{i}
\end{aligned}
$$

In this paper, the crisp linear fractional programming inventory model is formulated as

$$
\begin{aligned}
& \text { Maximize: } Z=\frac{\text { Total Profit }}{\text { Holding cost }} \\
& \text { Subject to, } \text { (i) Total Budget Limit, } \\
& \text { (ii) Storage Space Limit, } \\
& \text { (iii) Limit on Ordering Cost of Each Product. } \\
& \text { (iv) Non-Negative Restriction. }
\end{aligned}
$$

\section{Formulation of Constraints}

(i) Total Budget Limit: A budget constraint is all about the combinations of goods and services that a consumer may purchase given current prices within his or her given income. For the proposed model, the budget constraint is

$$
\sum_{i=1}^{n} P_{i} Q_{i} \leq B
$$

(ii) Storage Space Limit: $\sum_{i=1}^{n} f_{i} Q_{i} \leq W$

(iii) Limit on Ordering Cost of Each Product: We impose the upper limit of ordering cost as a constraint. Since $O C_{1}, O C_{2}, \ldots, O C_{n}$ are the ordering cost of $1^{\text {st }}$ product, $2^{\text {nd }}$ product, $\ldots, \mathrm{n}^{\text {th }}$ product, we can express the concerned constrained as follows:

$$
\begin{aligned}
& \text { For first product, } \quad \frac{\lambda D_{1}}{Q_{1}} \leq O C_{1} \\
& \Rightarrow \lambda D_{1}-O C_{1} \cdot Q_{1} \leq 0 \\
& \text { For second product, } \frac{\lambda D_{2}}{Q_{2}} \leq O C_{2} \\
& \Rightarrow \quad \lambda D_{2}-O C_{2} \cdot Q_{2} \leq 0 \\
& \text {, for } \mathrm{n}^{\text {th }} \text { product, } \lambda D_{n}-O C_{n} \cdot Q_{n} \leq 0 \\
& \Rightarrow \lambda m_{1} S_{n}^{-m_{2}}-O C_{n} \cdot Q_{n} \leq 0
\end{aligned}
$$

Similarly, for $\mathrm{n}^{\text {th }}$ product, $\lambda D_{n}-O C_{n} \cdot Q_{n} \leq 0$

(iv) Non-Negative Restriction:

$$
Q_{1}, Q_{2}, \ldots, Q_{i} \geq 0
$$

\section{Interval-VAlued Linear Fractional Programming INVENTORY PROBLEM}

In real life cases, it is observed that the selling price is uncertain due to dynamic behavior of the market. So it may be considered to vary in an interval. Let the selling price be represented by the interval $\left[S_{i L}, S_{i U}\right]$, where $S_{i L} \& S_{i U}$ are the left limit \& right limit of interval for $S_{i}$. Mathematically, we can express as

$$
S_{i}=\left[S_{i L}, S_{i U}\right], \text { with } S_{i L} \leq S_{i U}
$$


Operations Research and Applications : An International Journal (ORAJ), Vol.2, No.4, November 2015

Similarly, the purchasing, holding cost, and demand would be expressed as interval-valued numbers as

$$
\begin{aligned}
P_{i} & =\left[P_{i L}, P_{i U}\right], \text { with } P_{i L} \leq P_{i U} \\
h_{i} & =\left[h_{i L}, h_{i U}\right], \text { with } h_{i L} \leq h_{i U} \\
D_{i} & =\left[m_{1} S_{i L}^{-m_{2}}, m_{1} S_{i U}^{-m_{2}}\right], \text { with } m_{1} S_{i L}^{-m_{2}} \leq m_{1} S_{i U}^{-m_{2}}
\end{aligned}
$$

Accordingly, the constraints transform to the interval-valued form as follows

$$
\begin{aligned}
& \text { Total Budget Limit: } \quad \sum_{i=1}^{n}\left[P_{i L}, P_{i U}\right] Q_{i} \leq B \\
& \text { Storage Space Limit: } \quad \sum_{i=1}^{n} f_{i} Q_{i} \leq W \\
& \text { Limit on Ordering Cost of each Product: }
\end{aligned}
$$

For $\mathrm{n}^{\text {th }}$ product, we have

Non-Negative Restriction:

$$
\lambda\left[m_{1} S_{n L}^{-m_{2}}, m_{1} S_{n U}^{-m_{2}}\right] \leq Q_{n}\left[O C_{n L}, O C_{n U}\right]
$$

$$
Q_{1}, Q_{2}, \ldots, Q_{i} \geq 0
$$

In this case, we define the following interval-valued fuzzy linear fractional programming problem for the proposed inventory model,

$$
\begin{aligned}
& \text { (IVP1) Maximize: } \mathrm{Z}=\frac{\sum_{i=1}^{n}\left(\left[S_{i L}, S_{i U}\right]-\left[P_{i L}, P_{i U}\right]\right) Q_{i}}{\sum_{i=1}^{n} \frac{\left[h_{i L} h_{i U}\right] Q_{i}}{2}} \\
& \begin{aligned}
& \text { Subject to } \quad \sum_{i=1}^{n}\left[P_{i L}, P_{i U}\right] Q_{i} \leq B \\
& \sum_{i=1}^{n} f_{i} Q_{i} \leq W \\
& Q_{i}\left[O C_{i L}, O C_{i U}\right] \geq \lambda\left[m_{1} S_{n L}^{-m_{2}}, m_{1} S_{n U}^{-m_{2}}\right],(\text { for } \mathrm{i}=1,2, . ., \mathrm{n}) \\
& \text { and } Q_{i} \geq 0,(\text { for } \mathrm{i}=1,2, . ., \mathrm{n})
\end{aligned}
\end{aligned}
$$

Using the arithmetic operations of intervals, the interval-valued fractional objective function can be written as

$$
\begin{aligned}
Z & =\frac{\sum_{i=1}^{n}\left[S_{i L}-P_{i U}, s_{i U}-P_{i L}\right] Q_{i}}{\sum_{i=1}^{n}\left[\frac{h_{i L} Q_{i}}{2}, \frac{h_{i U} Q_{i L}}{2}\right]} \\
& =\frac{\left[\sum_{i=1}^{n}\left(S_{i L}-P_{i U}\right) Q_{i}, \sum_{i=1}^{n}\left(s_{i U}-P_{i L}\right) Q_{i}\right]}{\left[\sum_{i=1}^{n} \frac{h_{i L} Q_{i}}{2}, \sum_{i=1}^{n} \frac{h_{i U} Q_{i}}{2}\right]} \\
& =\left[\frac{\sum_{i=1}^{n}\left(s_{i L}-P_{i U}\right) Q_{i}}{\sum_{i=1}^{n} \frac{h_{i U} Q_{i}}{2}}, \frac{\sum_{i=1}^{n}\left(S_{i U}-P_{i L}\right) Q_{i}}{\sum_{i=1}^{n} \frac{h_{i L} Q_{i}}{2}}\right] \\
& =\left[Z_{L}\left(Q_{1}, Q_{2}, \ldots, Q_{n}\right), Z_{U}\left(Q_{1}, Q_{2}, \ldots, Q_{n}\right)\right]
\end{aligned}
$$

where $Z_{L}\left(Q_{1}, Q_{2}, \ldots, Q_{n}\right)=\frac{\sum_{i=1}^{n}\left(S_{i L}-P_{i U}\right) Q_{i}}{\sum_{i=1}^{n} \frac{h_{i U} Q_{i}}{2}}$

$$
\text { and } \quad Z_{U}\left(Q_{1}, Q_{2}, \ldots, Q_{n}\right)=\frac{\sum_{i=1}^{n}\left(S_{i U}-P_{i L}\right) Q_{i}}{\sum_{i=1}^{n} \frac{h_{i L} Q_{i}}{2}}
$$

Thus the single-objective function consisting of interval-valued number coefficients is transformed into multi-objective functions given by (27) where, in each objective function, the coefficients are crisp numbers. Hence, the interval-valued optimization problem (IVP1), transforms to following optimization problem as follows, 
Operations Research and Applications : An International Journal (ORAJ), Vol.2, No.4, November 2015

(IVP2) Maximize $\left[Z_{L}\left(Q_{1}, Q_{2}, \ldots, Q_{n}\right), Z_{U}\left(Q_{1}, Q_{2}, \ldots, Q_{n}\right)\right.$

Subject to $\sum_{i=1}^{n}\left[P_{i L}, P_{i U}\right] Q_{i} \leq B$,

$\sum_{i=1}^{n} f_{i} Q_{i} \leq W$,

$Q_{i}\left[O C_{i L}, O C_{i U}\right] \geq \lambda\left[m_{1} S_{i L}^{-m_{2}}, m_{1} S_{i U}^{-m_{2}}\right],($ for $\mathrm{i}=1,2, \ldots, \mathrm{n})$,

And $Q_{i} \geq 0$, (for $\mathrm{i}=1,2, . ., \mathrm{n}$ ).

To solution this problem, the techniques of classical linear fractional programming cannot be applied if and unless the above interval-valued structure of the problem be reduced into a standard linear fractional programming problem.

To deal with interval inequality constraints, we use the Tong's Approach [Tong (1994) \& Sengupta et al (2001)]. Tong deals with interval inequality constraints in a separate way.

According to Tong's Approach, each interval inequality constraint is transformed into $2^{n+1}$ crisp inequalities. Let us take a simple inequality constraint with a single variable:

$$
[10,20] x \leq[5,35] .
$$

According to Tong (1994), the interval inequality generates $2^{1+1}$ crisp inequalities:

$$
\begin{aligned}
& 10 x \leq 5 \Rightarrow x \leq 0.5 \\
& 10 x \leq 35 \Rightarrow x \leq 3.5 \\
& 20 x \leq 5 \Rightarrow x \leq 0.25 \\
& 20 x \leq 35 \Rightarrow x \leq 1.75
\end{aligned}
$$

Hence, the interval-valued linear fractional inventory problem (IVP2) can be transformed to a non-interval optimization problem as follows:

$$
\text { (IVP3) Maximize }\left[Z_{L}\left(Q_{1}, \ldots, Q_{n}\right), Z_{U}\left(Q_{1}, \ldots, Q_{n}\right)\right]
$$

where $Z_{L}\left(Q_{1}, Q_{2}, \ldots, Q_{n}\right)=\frac{\sum_{i=1}^{n}\left(S_{i L}-P_{i U}\right) Q_{i}}{\sum_{i=1}^{n} \frac{h_{i U} Q_{i}}{2}}$

Subject to: $\sum_{i=1}^{n} P_{i L} Q_{i} \leq B$

$$
Z_{U}\left(Q_{1}, Q_{2}, \ldots, Q_{n}\right)=\frac{\sum_{i=1}^{n}\left(S_{i U}-P_{i L}\right) Q_{i}}{\sum_{i=1}^{n} \frac{h_{i L} Q_{i}}{2}}
$$

$$
\begin{aligned}
\sum_{i=1}^{n} P_{i U} Q_{i} & \leq B \\
\sum_{i=1}^{n} f_{i} Q_{i} & \leq W \\
O C_{i L} Q_{i} & \geq \lambda m_{1} S_{i L}^{-m_{2}},(\text { for } \mathrm{i}=1,2, \ldots, \mathrm{n}) \\
O C_{i U} Q_{i} & \geq \lambda m_{1} S_{i L}^{-m_{2}},(\text { for } \mathrm{i}=1,2, . ., \mathrm{n}) \\
O C_{i L} Q_{i} & \geq \lambda m_{1} S_{i U}^{-m_{2}},(\text { for } \mathrm{i}=1,2, . ., \mathrm{n}) \\
O C_{i U} Q_{i} & \geq \lambda m_{1} S_{i U}^{-m_{2}},(\text { for } \mathrm{i}=1,2, . ., \mathrm{n}) \\
Q_{i} & \geq 0,(\text { for } \mathrm{i}=1,2, . ., \mathrm{n})
\end{aligned}
$$

Now, let us write the corresponding optimization problem of (IVP3) as follows:

(IVP4) Maximize $\mathrm{g}(\mathrm{x})=Z_{L}\left(Q_{1}, Q_{2}, \ldots, Q_{n}\right)+Z_{U}\left(Q_{1}, Q_{2}, \ldots, Q_{n}\right)$ 
Operations Research and Applications : An International Journal (ORAJ), Vol.2, No.4, November 2015

$$
\begin{array}{ll}
\text { Subject to } & \sum_{i=1}^{n} P_{i L} Q_{i} \leq B \\
& \sum_{i=1}^{n} P_{i U} Q_{i} \leq B \\
& \sum_{i=1}^{n} f_{i} Q_{i} \leq W \\
O C_{i L} Q_{i} \geq \lambda m_{1} S_{i L}^{-m_{2}},(\text { for } \mathrm{i}=1,2, \ldots, \mathrm{n}) \\
O C_{i U} Q_{i} \geq \lambda m_{1} S_{i L}^{-m_{2}},(\text { for } \mathrm{i}=1,2, \ldots, \mathrm{n}) \\
O C_{i L} Q_{i} \geq \lambda m_{1} S_{i U}^{-m_{2}},(\text { for } \mathrm{i}=1,2, \ldots, \mathrm{n}) \\
O C_{i U} Q_{i} \geq \lambda m_{1} S_{i U}^{-m_{2}},(\text { for } \mathrm{i}=1,2, \ldots, \mathrm{n}) \\
Q_{i} \geq 0,(\text { for } \mathrm{i}=1,2, \ldots, \mathrm{n})
\end{array}
$$

To solve the (IVP3), theorem (Wu (2008)) is conveniently used. According to this theorem, the non-dominated solution of (IVP3), would be determined by solving its corresponding optimization problem (IVP4).

\section{NUMERICAL EXAMPLES}

To illustrate the proposed approach, we solve the following two numerical examples in crisp and interval-value cases:

Example1. Crisp Case Consider an inventory model with following input data (in proper units):

Table 1: Input data (Crisp Case)

\begin{tabular}{|c|l|l|l|l|l|l|l|l|l|c|}
\hline Product & $h_{i}$ & $P_{i}$ & $S_{i}$ & $O C_{i}$ & $f_{i}$ & $m_{1}$ & $m_{2}$ & $\lambda$ & W & $B$ \\
\hline $\mathrm{i}=1$ & 12 & 125 & 200 & 80 & 2 & & & & & \\
\cline { 1 - 6 } $\mathrm{i}=2$ & 16 & 160 & 220 & 90 & 4 & 80,000 & 1.20 & 7 & 300 & 90,000 \\
\hline
\end{tabular}

\begin{tabular}{|c|c|c|c|c|c|c|c|c|c|c|}
\hline Product & $h_{i}$ & $P_{i}$ & $S_{i}$ & $O C_{i}$ & $f_{i}$ & $m_{1}$ & $m_{z}$ & $\lambda$ & W & $\bar{B}$ \\
\hline$i=1$ & {$[10,14]$} & {$[120,130]$} & {$[190,210]$} & {$[70,90]$} & 2 & & & & & \\
\hline$i=2$ & {$[14,18]$} & {$[150,170]$} & {$[210,230]$} & {$[80,100]$} & 4 & 80,000 & 1.20 & 7 & 300 & 90,000 \\
\hline
\end{tabular}

Maximize: $\quad Z=\frac{\sum_{i=1}^{2}\left(S_{i}-P_{i}\right) Q_{i}}{\sum_{i=1}^{2} \frac{n_{i} Q_{i}}{2}}=\frac{75 Q_{1}+60 Q_{2}}{6 Q_{1}+8 Q_{2}}$

Subject to: $\sum_{i=1}^{n} P_{i} Q_{i} \leq B \quad \Rightarrow 125 Q_{1}+160 Q_{2} \leq 90000$,

$$
\begin{array}{ll}
\sum_{i=1}^{n} f_{i} Q_{i} \leq W & \Rightarrow 2 Q_{1}+4 Q_{2} \leq 300, \\
O C_{n} \cdot Q_{n} \geq \lambda m_{1} S_{n}^{-m_{2}} & \Rightarrow 80 Q_{1} \geq 970.4027, \text { and } 90 Q_{2} \geq 865.5273 .
\end{array}
$$

The optimal solution is $Q_{1}^{*}=130.7658, Q_{2}^{*}=9.6170, Z^{*}=12.0535$,

Maximum profit $=10384.455$.

Interval-Values Case Consider an inventory model with following input data:

Table 2: Input data (Interval-Valued Case) 
Operations Research and Applications : An International Journal (ORAJ), Vol.2, No.4, November 2015

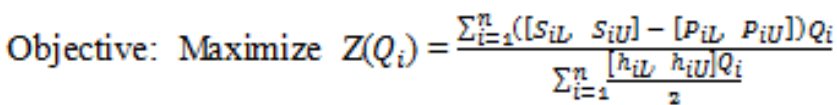

$$
\begin{aligned}
& =\frac{([190,210]-[120,130]) Q_{1}+([210,230]-[150,170]) Q_{2}}{\frac{[10,14] Q_{1}}{2}+\frac{[14,18] Q_{2}}{2}}, \\
& Z_{L}\left(Q_{1}, Q_{2}, \ldots, Q_{n}\right)=\frac{\sum_{i=1}^{n}\left(s_{i L}-P_{i} U\right) Q_{i}}{\sum_{i=1}^{n} \frac{n_{i U} Q_{i}}{2}}=\frac{(190-130) Q_{1}+(210-170) Q_{2}}{7 Q_{1}+9 Q_{2}}=\frac{60 Q_{1}+40 Q_{2}}{7 Q_{1}+9 Q_{2}} \\
& Z_{U}\left(Q_{1}, Q_{2}, \ldots, Q_{n}\right)=\frac{\sum_{i=1}^{n}\left(S_{i U}-P_{i}\right) Q_{i}}{\sum_{i=1}^{n} \frac{n_{i L} Q_{i}}{2}}=\frac{(210-120) Q_{1}+(230-150) Q_{2}}{5 Q_{1}+7 Q_{2}}=\frac{90 Q_{1}+80 Q_{2}}{5 Q_{1}+7 Q_{2}} .
\end{aligned}
$$

The corresponding optimization problem would be:

$$
\begin{aligned}
& \text { Maximize } g(\mathrm{x})=\frac{60 Q_{1}+40 Q_{2}}{7 Q_{1}+9 Q_{2}}+\frac{90 Q_{1}+80 Q_{2}}{5 Q_{1}+7 Q_{2}}, \\
& \text { Subject to: } \sum_{i=1}^{n} P_{i L} Q_{i} \leq B \quad \Rightarrow 120 Q_{1}+150 Q_{2} \leq 90000 \text {, } \\
& \sum_{i=1}^{n} P_{i U} Q_{i} \leq B \quad \Rightarrow 130 Q_{1}+170 Q_{2} \leq 90000 \text {, } \\
& \sum_{i=1}^{n} f_{i} Q_{i} \leq W \quad \Rightarrow 2 Q_{1}+4 Q_{2} \leq 300 \text {, } \\
& O C_{i L} Q_{i} \geq \lambda m_{1} S_{i L}^{-m_{2}} \quad \Rightarrow 70 Q_{1} \geq 1032.0095,80 Q_{2} \geq 915.2187 \text {, } \\
& O C_{i U} Q_{i} \geq \lambda m_{1} S_{i L}^{-m_{2}} \Rightarrow 90 Q_{1} \geq 1032.0095,100 Q_{2} \geq 915.2187 \text {, } \\
& O C_{i L} Q_{i} \geq \lambda m_{1} S_{i U}^{-m_{2}} \quad \Rightarrow 70 Q_{1} \geq 915.2187, \quad 80 Q_{2} \geq 820.5681 \text {, } \\
& O C_{i U} Q_{n} \geq \lambda m_{1} S_{i U}^{-m_{2}} \quad \Rightarrow 90 Q_{1} \geq 915.2187,100 Q_{2} \geq 820.5681 \\
& \text { and } Q_{1} \geq 0, Q_{2} \geq 0
\end{aligned}
$$

The optimal solution is $Q_{1}^{*}=127.1197, Q_{2}^{*}=11.4402$ with optimum value $g\left(Q_{1}^{*}, Q_{2}^{*}\right)=25.4081$.

Maximum Profit $=([190,210]-[120,130]) Q_{1}^{*}+([210,230]-[150,170]) Q_{2}^{*}$

$$
\begin{aligned}
& =[60,90] Q_{1}^{*}+\left([40,80] Q_{2}^{*}\right. \\
& =\left[60 Q_{1}^{*}+40 Q_{2}^{*}, 90 Q_{1}^{*}+80 Q_{2}^{*}\right] \\
& =[8084.79,12355.989] .
\end{aligned}
$$

Hence, the bounds of profit are 8084.79 (lower bound), and 12355.989 (upper bound).

Example2. Crisp Case Consider an inventory model with following input data (in proper units):

Table 3: Input data (Crisp Case)

\begin{tabular}{|l|l|l|l|l|l|l|l|l|l|l|}
\hline Product & $h_{\mathrm{i}}$ & $P_{\mathrm{i}}$ & $S_{i}$ & $O C_{\mathrm{i}}$ & $f_{\mathrm{i}}$ & $m_{1}$ & $m_{2}$ & $\lambda$ & $\mathrm{W}$ & $B$ \\
\hline $\mathrm{i}=1$ & 12 & 125 & 180 & 70 & 1 & & & & & \\
\cline { 1 - 3 } $\mathrm{i}=2$ & 15 & 150 & 220 & 80 & 2 & 15,000 & 1.10 & $\mathbf{5}$ & 400 & 60,000 \\
\hline
\end{tabular}

$$
\begin{aligned}
& \text { Maximize: } \quad Z=\frac{\sum_{i=1}^{n}\left(S_{i}-P_{i}\right) Q_{i}}{\sum_{i=1}^{n} \frac{n_{i} Q_{i}}{2}}=\frac{55 Q_{1}+70 Q_{2}}{6 Q_{1}+7.5 Q_{2}} \\
& \text { Subject to: } \sum_{i=1}^{n} P_{i} Q_{i} \leq B \quad \Rightarrow 125 Q_{1}+150 Q_{2} \leq 60000 \text {, } \\
& \sum_{i=1}^{n} f_{i} Q_{i} \leq W \quad \Rightarrow Q_{1}+2 Q_{2} \leq 400 \text {, } \\
& O C_{n} \cdot Q_{n} \geq \lambda m_{1} S_{n}^{-m_{2}} \Rightarrow 70 Q_{1} \geq 247.8914 \text {, and } 80 Q_{2} \geq 198.7908 \text {. }
\end{aligned}
$$

The optimal solution is $Q_{1}^{*}=3.5413, Q_{2}^{*}=198.2293, Z^{*}=9.3310$, Maximum profit $=14070.8225$. 
Operations Research and Applications : An International Journal (ORAJ), Vol.2, No.4, November 2015

Interval-Values Case Consider an inventory model with following input data:

Table 4: Input data (Interval-Valued Case)

\begin{tabular}{|c|c|c|c|c|c|c|c|c|c|c|}
\hline Product & $h_{\mathrm{i}}$ & $P_{i}$ & $S_{i}$ & $O C_{i}$ & $f_{i}$ & $m_{1}$ & $m_{2}$ & $\lambda$ & $\mathrm{W}$ & $B$ \\
\hline $\mathrm{i}=1$ & {$[8,16]$} & {$[100,150]$} & {$[160,200]$} & {$[60,80]$} & 1 & & & & & \\
\hline$i=2$ & {$[12,18]$} & {$[125,175]$} & {$[200,240]$} & {$[70,90]$} & 2 & 15,000 & 1.10 & 5 & 400 & 60,000 \\
\hline
\end{tabular}

Objective: Maximize $\mathrm{Z}\left(Q_{i}\right)=\frac{\sum_{i=1}^{n}\left(\left[s_{i L} s_{i U}\right]-\left[p_{i L}, p_{i U}\right]\right) Q_{i}}{\sum_{i=1}^{n} \frac{\left[h_{i L}, h_{i U}\right] Q_{i}}{2}}$

$$
\begin{gathered}
=\frac{([160,200]-[100,150]) Q_{1}+([200,240]-[125,175]) Q_{2}}{\frac{[8,16] Q_{1}}{2}+\frac{[12,18] Q_{2}}{2}}, \\
Z_{L}\left(Q_{1}, Q_{2}, \ldots, Q_{n}\right)=\frac{\sum_{i=1}^{n}\left(S_{i L}-P_{i}\right) Q_{i}}{\sum_{i=1}^{n} \frac{h_{i U} Q_{i}}{2}}=\frac{(160-150) Q_{1}+(200-175) Q_{2}}{8 Q_{1}+9 Q_{2}}=\frac{10 Q_{1}+25 Q_{2}}{8 Q_{1}+9 Q_{2}} \\
Z_{U}\left(Q_{1}, Q_{2}, \ldots, Q_{n}\right)=\frac{\sum_{i=1}^{n}\left(S_{i U}-P_{i}\right) Q_{i}}{\sum_{i=1}^{n} \frac{n_{i L} Q_{i}}{2}}=\frac{(200-100) Q_{1}+(240-125) Q_{2}}{4 Q_{1}+6 Q_{2}}=\frac{100 Q_{1}+115 Q_{2}}{4 Q_{1}+6 Q_{2}} .
\end{gathered}
$$

The corresponding optimization problem would be:

$$
\begin{aligned}
& \text { Maximize } g(\mathrm{x})=\frac{10 Q_{1}+25 Q_{2}}{8 Q_{1}+9 Q_{2}}+\frac{100 Q_{1}+115 Q_{2}}{4 Q_{1}+6 Q_{2}} \text {, } \\
& \text { Subject to: } \sum_{i=1}^{n} P_{i L} Q_{i} \leq B \quad \Rightarrow 100 Q_{1}+125 Q_{2} \leq 60000 \text {, } \\
& \sum_{i=1}^{n} P_{i U} Q_{i} \leq B \quad \Rightarrow 150 Q_{1}+175 Q_{2} \leq 60000 \text {, } \\
& \sum_{i=1}^{n} f_{i} Q_{i} \leq W \quad \Rightarrow Q_{1}+2 Q_{2} \leq 400 \text {, } \\
& O C_{n L} Q_{n} \geq \lambda m_{1} S_{i L}^{-m_{2}} \Rightarrow 60 Q_{1} \geq 282.1819,70 Q_{2} \geq 220.7640 \text {, } \\
& O C_{n U} Q_{n} \geq \lambda m_{1} S_{i L}^{-m_{2}} \quad \Rightarrow 80 Q_{1} \geq 282.1819,90 Q_{2} \geq 220.7640 \text {, } \\
& O C_{n L} Q_{n} \geq \lambda m_{1} S_{i U}^{-m_{2}} \Rightarrow 60 Q_{1} \geq 220.7640,70 Q_{2} \geq 180.6462 \text {, } \\
& O C_{n U} Q_{n} \geq \lambda m_{1} S_{i U}^{-m_{2}} \Rightarrow 80 Q_{1} \geq 220.7640,90 Q_{2} \geq 180.6462 \text {, } \\
& \text { and } Q_{1} \geq 0, Q_{2} \geq 0 \text {. }
\end{aligned}
$$

The optimal solution is $Q_{1}^{*}=393.6925, Q_{2}^{*}=3.1538$ with optimum value $g\left(Q_{1}^{*}, Q_{2}^{*}\right)=26.1944$.

Maximum Profit $=\left(([160,200]-[100,150]) Q_{1}^{*}+([200,240]-[125,175]) Q_{2}^{*}\right)$

$$
\begin{aligned}
& =[10,100] Q_{1}^{*}+\left([75,115] Q_{2}^{*}\right. \\
& =\left[10 Q_{1}^{*}+75 Q_{2}^{*}, 100 Q_{1}^{*}+115 Q_{2}^{*}\right] \\
& =[4173.46,39731.937] .
\end{aligned}
$$

Hence, the bounds of profit are 4173.46 (lower bound), and 39731.937 (upper bound).

\section{CONClusions}

In this paper, the author presented an approach to solve interval-valued inventory optimization problem based on linear fractional programming. The uncertainty in inventory parameters is represented by interval-valued numbers. Using interval arithmetic, the interval-valued optimization problem is changed into a crisp multi-objective linear fractional programming problem. LINGO package is used to solve the subsequent optimization problem. The optimal order quantity and profit bounds are determined. 
Operations Research and Applications : An International Journal (ORAJ), Vol.2, No.4, November 2015

For future directions, the paper can be extended to inventory model with shortages case and inventory model with price-discount. Moreover, it can also be extended to interval-valued multiobjective optimization case.

\section{REFERENCES}

[1] Alefeld, G., Herzberger, J., (1983), Introduction to interval computations. Academic Press, London.

[2] Bhurjee, A.K., Panda, G., (2012), Efficient solution of interval optimization problem. Mathematical Methods of Operation Research, 76(3), 273-288.

[3] Carla Oliveira, Carlos Henggeler Antunes, (2007), Multiple objective linear programming models with interval coefficients - an illustrated overview. European Journal of Operational Research, 181(3), 1434-1463.

[4] Chanas, S., Kuchta, D., (1996), Multi-objective programming in optimization of interval objective functions-a generalized approach. European Journal of Operational Research, 94(3), 594-598.

[5] Charnes, A., Cooper, W.W., (1962), Programming with linear fractional functions. Naval Research Logistics. Quarterly, 9(3-4), 181-186.

[6] Charnes, A., Granot, F., Phillips, F., (1977), An algorithm for solving interval linear programming problems. Operations Research, 25(4), 688-695.

[7] Chinneck, J.W., Ramadan, K., (2000), Linear programming with interval coefficients. Journal of Operational Research Society, 51(2), 209-220

[8] Deepmala, (2014), A Study on Fixed Point Theorems for Nonlinear Contractions and its Applications, Ph.D. Thesis, Pt. Ravishankar Shukla University, Raipur (Chhatisgarh) India - 492010.

[9] Effati, S., Pakdaman, M., (2012), Solving the interval-valued linear fractional programming problem. American Journal of Computational Mathematics, 2(1), 51-55.

[10] Ishibuchi, H.,Tanaka, H., (1990), Multi objective programming in optimization of the interval objective function. European Journal of Operational Research, 48(2), 219-225.

[11] Kumar, P., Dutta, D., (2015), Multi-objective Linear Fractional Inventory Model of Multi-Products with Price-Dependant Demand Rate in Fuzzy Environment, International Journal of Mathematics in Operations Research : Inderscience Journal, 7(5), 547-565.

[12] Levin, V.I., (1999), Nonlinear optimization under interval uncertainty. Cybernetics and Systems Analysis, 35(2), 297-306.

[13] Liu, S.T., (2006), Computational method for the profit bounds of inventory model with interval demand and unit cost. Applied Mathematics and Computation, 183(1), 499-507.

[14] Liu, S.T., Wang, R.T., (2007), A numerical solution method to interval quadratic programming. Applied Mathematics and Computation, 189(2), 1274-1281.

[15] Liu, S.T., (2008), Posynomial geometric programming with interval exponents and coefficients. European Journal of Operational Research, 186(1), 17-27.

[16] Mishra, V. N., (2007), Some Problems on Approximations of Functions in Banach Spaces, Ph.D. Thesis, Indian Institute of Technology, Roorkee - 247 667, Uttarakhand, India.

[17] Moore, R., Lodwick, W., (2003), Interval analysis and fuzzy set theory. Fuzzy Sets and Systems, 135(1), 5-9.

[18] Mráz, F., (1998), Calculating the exact bounds of optimal values in LP with interval coefficients. Annals of Operation Research, 81(1), 51-62.

[19] Sadjadi, S.J., Aryanezhad, M.B., Sarfaraj, A., (2005), A fuzzy approach to solve a multi-objective linear fractional inventory model. Journal of Industrial Engineering International, 1(1), 43-47.

[20] Sengupta, A., Pal, T.K., Chakraborty, D., (2001), Interpretation of inequality constraints involving interval coefficients and a solution to interval linear programming. Fuzzy Sets and Systems, 119(1), 129-138.

[21] Steuer, R.E., (1981), Algorithms for linear programming problems with interval objective function coefficients. Mathematics of Operation Research, 6(3), 333-348.

[22] Toksarı, M.D., (2008), Taylor series approach to fuzzy multi objective linear fractional programming. Information Sciences, 178(4), 1189-1204.

[23] Tong, S., (1994), Interval number and fuzzy number linear programming. Fuzzy Sets and Systems, 66(3), 301-306.

[24] Vandana., Sharma, B.K., (2015), An EPQ inventory model for non-instantaneous deteriorating item under trade credit policy, International Journal of Mathematical Science \& Engineering Applications, (IJMSEA), 9(1), 179-188. 
Operations Research and Applications : An International Journal (ORAJ), Vol.2, No.4, November 2015

[25] Wu, H.C., (2007), The Karush-Kuhn-Tucker optimality conditions in an optimization problem with interval-valued objective function. European Journal of Operational Research, 176(1), 46-59.

[26] Wu, H.C., (2008a), On interval-valued nonlinear programming problems. Journal of Mathematical Analysis and Applications, 338(1), 299-316.

[27] Wu, H.C., (2008b), Wolfe duality for interval-valued optimization. Journal of Optimization Theory and Applications, 138(3), 497-509.

[28] Wu, H.C., (2010), Duality theory for optimization problems with interval-valued objective functions. Journal of Optimization Theory and Applications, 144(3), 615-628. 